\title{
Pitch angle distribution of trapped energetic protons and helium isotope nuclei measured along the Resurs-01 No. 4 LEO satellite
}

\author{
A. Leonov ${ }^{1,2}$, M. Cyamukungu ${ }^{1}$, J. Cabrera ${ }^{1}$, P. Leleux ${ }^{1}$, J. Lemaire ${ }^{1}$, G. Gregorie ${ }^{1}$, S. Benck ${ }^{1}$, V. Mikhailov ${ }^{2}$,

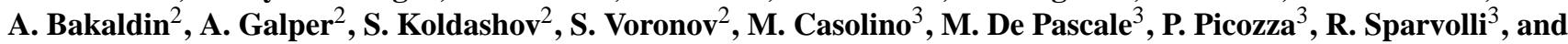 \\ M. Ricci ${ }^{4}$ \\ ${ }^{1}$ Center for Space Radiations (CSR); Chemin du Cyclotron 2, 1348 Louvain-la-Neuve, Belgium \\ ${ }^{2}$ Moscow Engineering PHysics Institute (MEPHI), state university; Kashirskoe shosse 31, 115409 Moscow, Russia \\ ${ }^{3}$ Istituto Nazionale di Fisica Nucleare sezione di Roma2, University of Rome "Tor Vergata", I-00133 Rome, Italy \\ ${ }^{4}$ Istituto Nazionale di Fisica Nucleare, Laboratori Nazionali di Frascati, Via Enrico Fermi 40, I-00044 Frascati, Italy
}

Received: 8 February 2005 - Revised: 25 April 2005 - Accepted: 6 May 2005 - Published: 22 November 2005

Part of Special Issue "1st European Space Weather Week (ESWW)"

\begin{abstract}
The NINA detector on board the Resurs-01 No. 4 satellite $\left(835 \mathrm{~km}, 98^{\circ}\right.$ inclination) is equipped with particle trackers based on silicon strip detectors. From the energy deposited in each of its silicon layers the mass, the momentum direction and energy of incident particles have been determined. The resolutions in mass and energy allow identification of $\mathrm{H}$ and $\mathrm{He}$ isotopes over the $10-50 \mathrm{MeV} / \mathrm{n}$ energy range. The angular resolution is about $2.5^{\circ}$. We present the direct measurements of proton and helium isotopes pitch angle distributions derived from Resurs-01 No.4/NINA observations and their variations as functions of $(\mathrm{B}, \mathrm{L})$ coordinates and energy. The measurements of trapped helium isotopes spectrum are also presented.
\end{abstract}

Keywords. Interplanetary physics (Cosmic rays) - Magnetospheric physics (Energetic particles, trapped) - Solar physics, astrophysics, and astronomy (Instruments and techniques)

\section{Introduction}

Besides the energy spectra, angular distributions of protons at low altitudes are needed to accurately evaluate their effects on space crew and equipments. However, detectors for direct angular and energy distribution measurements may be of a complexity level and a mass budget that are scarcely allowed on small satellite missions. The New Instrument for Nuclear Analysis (NINA) instrument, accommodated on the Resurs-01 No.4 satellite, is that type of complex and heavy telescope. Data acquired using such a detector usually serve

Correspondence to: A. Leonov

(AlexeyAnatolievich@rambler.ru) for calibration of simple instruments, which perform indirect angle and energy distributions measurements. Cyamukungu et al. (2001) have described methods and conditions to completely but indirectly determine the angular distributions of particle fluxes over a telescope field of view, for three-axis stabilised satellites. Similarly, the indirect measurement of particle pitch angle distributions on a spinning satellite has been described in Auschrat and Fischer (1974). NINA data may also be used to validate models of angular distribution (Armstrong et al. (1990); Badhwar and Konradi (1990)) in different solar activity and geomagnetic conditions.

In this paper, we present the angular distribution of protons and helium isotope nuclei measured by NINA, along with its variation as functions of $(\mathrm{B}, \mathrm{L})$ coordinates and energy. The description of the NINA instrument, orbit operations and detector performance in orbit may be found in Bidoli et al. (2001). For clarity, it is summarized in Sects. 2 and 3. The off-line data processing methods are also described in Bidoli et al. (2001). In Sect. 4, the flux measurement is described along with the results and comparisons with available data. In conclusion, the recommendations for the design of directional particle telescopes to study angle distributions of trapped particles are also presented.

\section{The instrument}

The instrument NINA is a stack of 16 planes, each of which comprises two $6 \times 6 \mathrm{~cm}^{2}$ n-type silicon detectors segmented into 16 strips. The two silicon detectors are perpendicular to each other, so as to provide the $\mathrm{X}$ and $\mathrm{Y}$ coordinate information of the particle track. The thickness of the first two 
detectors is $150 \mu \mathrm{m}$, while the thickness of all the others is $380 \mu \mathrm{m}$, for a total of $11.7 \mathrm{~mm}$ of active silicon.

The inter-planar distance is $14 \mathrm{~mm}$, but the first and second planes are separated by $85 \mathrm{~mm}$, for a better measurement of the particle incidence angle. The angular resolution is about $2.5^{\circ}$. The total telescope height is $29.5 \mathrm{~cm}$ for a total mass of $40 \mathrm{~kg}$ and $40 \mathrm{~W}$ of power consumption. A $300 \mu / \mathrm{m}$ thick aluminum foil covers the detector aperture.

The main trigger of the data acquisition system requires a particle to reach at least the first layer (X) of the second detector plane. A veto system, ensuring the recording of particles entering the detector from above only, is implemented by setting in anticoincidence strips 1 and 16 of planes 2-15 (lateral anticoincidence) and all strips of plane 16 (bottom anticoincidence). The optimal performance of NINA is achieved by requesting the full containment of the particle inside the detector. The request of containment inside the telescope defines the upper energy limits for particle detection, which is equal to about $50 \mathrm{MeV} / \mathrm{n}$ for ${ }^{4} \mathrm{He}$. The energy resolution is $\sim 1 \mathrm{MeV}$.

The instrument has two different modes of particle detection: the high-threshold (HT) mode used to detect the nuclei from helium to iron in the full energy range (10-200 MeV/n) and the low-threshold (LT) that allows one to also detect hydrogen from 10 to $50 \mathrm{Mev} / \mathrm{n}$. In the high-threshold (HT) mode it was possible to detect hydrogen isotopes only in a narrow energy range (11-16 MeV for $\left.{ }^{1} \mathrm{H}\right)$. More than $95 \%$ of the Resurs-01 No.4/NINA mission measurements were carried out in HT mode to avoid a memory saturation that would occur due to the very high proton flux in the radiation belt.

\section{Detector performance in orbit}

The container with the sensitive part of the NINA detector was mounted on the top side external to the Resurs-01 No.4 satellite, in such a way as to always point upward in the radial direction during the flight. The satellite was launched on 10 July 1998 in a near-Earth polar orbit with inclination $98^{\circ}$ and altitude $835 \mathrm{~km}$. It made $\sim 14$ revolutions per day, and when the orbit was crossing the SAA ( $\sim 7$ times per day), NINA could measure particles trapped in the inner radiation belt. The period of observation, taken under consideration, ranges from November 1998 to April 1999. The total lifetime for this period is $6.3 \times 10^{6} \mathrm{~s}$, while inner radiation belt measurements for $\mathrm{L}$-shell $<1.3$ cover approximately $5 \%$ of the whole exposure time. During the period of measurements, $\sim 10^{5}$ protons and $\sim 10^{3}$ helium nuclei were identified in the inner radiation belt. Data also include particles collected during solar energetic particle events, because even in conditions of intense solar activity, the fluxes measured by NINA in the inner radiation belt remained practically constant. The background evaluation and systematic errors estimation for events detected by the NINA instrument were presented in Bakaldin et al. (2002a) and Bidoli et al. (2001), respectively. The intensity contribution of background events and systematic errors does not exceed several percents.

\section{Measurements of trapped particles}

To calculate the pitch angle of a detected trapped particle it is necessary that this particle reach at least the second layer (Y) of the second detector plane. In this case two $\mathrm{X}$ and two $Y$ coordinates allow to define the entrance angle of the particle and then, using the orientation angle $\gamma$ (angle between the detector axis and the magnetic field direction), it is possible to calculate the pitch angle. Unfortunately, in HT NINA mode it is possible to reliably detect protons which hit more than 3 layers, corresponding only to the $12-15 \mathrm{MeV}$ energy interval.

As the fluxes of trapped particles in the inner radiation belt is a rapidly varying function of the $(\mathrm{B}, \mathrm{L})$ coordinates (Fischer et al., 1977), we divided all our inner radiation belt data into bins for available $\mathrm{L}$ and $\mathrm{B}$ values in a 0.01 step for both coordinates. Then, in order to take into account the dependence of the experimental count rate on the orientation angle $\gamma$ (through the variation of the efficiency), we also introduced $\gamma$-bins with a $5^{\circ}$ step for each (B, L) bin and calculated the observation time for every final bin. So, the fluxes were obtained in the same ranges in $(\mathrm{L}, \mathrm{B})$ coordinates.

The counting rates were converted into directional fluxes using the detector orientation and pitch angle-dependent efficiencies obtained by means of the Monte Carlo simulations based on the CERN-GEANT code (Brun et al., 1994).

\subsection{Pitch angle distributions of trapped protons}

Two methods were used to obtain the angular distributions of trapped protons: In the first one, particles entering the instrument within a $5^{\circ}$ F.O.V. half angle, relative to the device axis, are assumed to have a pitch angle equal to the orientation angle $\gamma$. This orientation (pitch) angle is directly deduced using the IGRF 1995 (epoch 1998) magnetic field model. The flux of such particles is measured as a function of the orientation angle $\gamma$ using the easily calculated efficiency of perpendicularly incident particles for a given $(B, L)$ bin. Figure 1a shows the fluxes for different $(B, L)$ bins derived by use of the input data described above.

The geographical position where the fluxes were measured is shown in Fig. 1b. Because of the limited volume of device memory and the high count rates in the core of the SAA, the single strip event information was not recorded by the instrument data acquisition system, if the minute averaging counting rate had become more than $10 \mathrm{~Hz}$ (Bidoli et al., 2001). So, the inner radiation belt data were available only on the boundary. It is important to note that the influence of the device saturation effect in the SAA is not essential in the NINA experiment due to the high time resolution of the trigger system $(2 \mu \mathrm{s})$. The respective dead time and the time required by the readout system were accounted for in exposure time calculations (Bakaldin et al., 2002b). Because of poor statistics, due to the small solid angle in the limited $5^{\circ}$ F.O.V angle, we have combined several L-bins and assumed that the angular distribution is symmetrical about the plane perpendicular to 


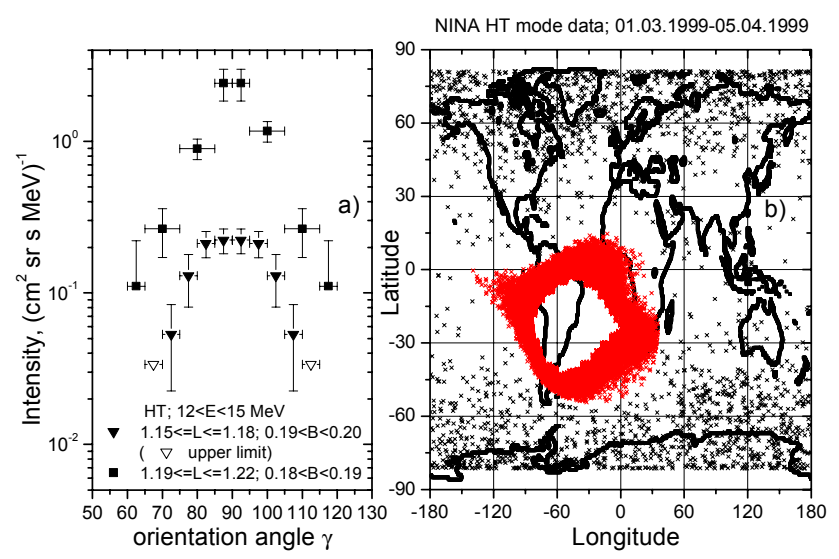

Fig. 1. (a) Pitch angle distributions of $12<\mathrm{E}<15 \mathrm{MeV}$ protons impinging NINA from a radial direction for different $(\mathrm{B}, \mathrm{L})$ bins. (b) Geographical positions where the pitch angle distributions in panel (a) have been measured (red signs).

the magnetic field vector, and combined events with symmetrical pitch angles.

In the second method, particles with incidence angles greater than $5^{\circ}$ with respect to the detector axis were included. The obtained equator pitch angle distributions for different L-shells that take into account a larger F.O.V. angle are shown in Fig. 2a. Figure 2b shows an example of the calculated instrument geometrical factor per $5^{\circ}$ pitch angle interval as a function of proton pitch angle for the orientation angle $\gamma=87.5^{\circ}$, for the energy range $12-15 \mathrm{MeV}$, which was used to reconstruct the fluxes. The isotropic geometrical factor for those protons is also shown.

We defined the exponent $\mathrm{n}$ of the pitch angle distribution by applying the method proposed in Fischer et al. (1977). In this method the power dependence between the experimental counting rates of trapped protons and the geomagnetic latitude $\left(\mathrm{B} / \mathrm{B}_{0}\right)$ for a given $\mathrm{L}$-shell is used. If the counting rates which are received from identical experimental orientations are plotted as a function of geomagnetic latitude in a bilogarithmic plot, the slope of the resulting straight line determines the exponent $\mathrm{n}$. Such a dependence of proton counting rates on $\mathrm{B} / \mathrm{B}_{0}$ values for $\mathrm{L}=1.24$ is shown in Fig. 3a. Near the equator there is a linear increase in the counting rates, which defines the slope, which is related to the exponent $\mathrm{n}$. Then a short zone of transition ends in the very steep slope near the loss cone. Only three points in Fig. 3a have to be used to deduce the exponent $n$, because near the loss cone this dependence becomes nonlinear, and then the slope is not constant for this part of the curve and exponent $\mathrm{n}$ varies (Fischer et al., 1977).

The variation of the $n$ value as a function of $L$, obtained from the NINA data, is presented in Fig. 3b. The results deduced from the S-30 experiment on board the DIAL satellite (Fischer et al., 1977) are also shown. There is a good agreement between NINA and S-30 results. The NINA and S-30 data were collected during the maximum of the solar cycle. So it appears normal that the gradient of the atmospheric

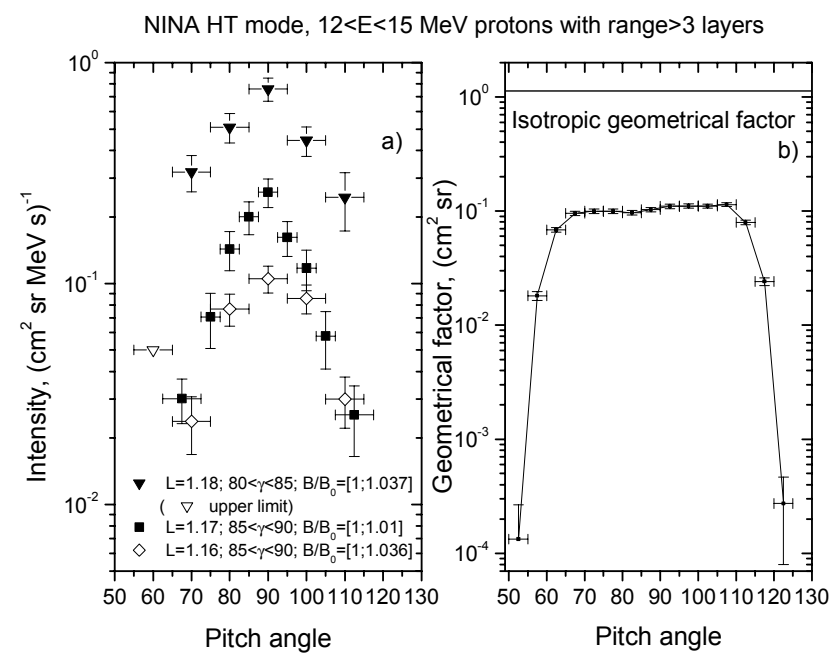

Fig. 2. (a) Equator pitch angle distribution of $12<\mathrm{E}<15 \mathrm{MeV}$ protons with a range more than 3 views impinging NINA from the whole F.O.V. for different L-shells (width of each L-bin is 0.01). (b) Geometrical factor as a function of a proton's pitch angle for orientation angle $\gamma=87.5^{\circ}$, for a pitch angle step $5^{\circ}$ and for the energy range $12-15 \mathrm{MeV}$, which was used to reconstruct the fluxes. The isotropic geometrical factor for those protons is also shown (solid line).
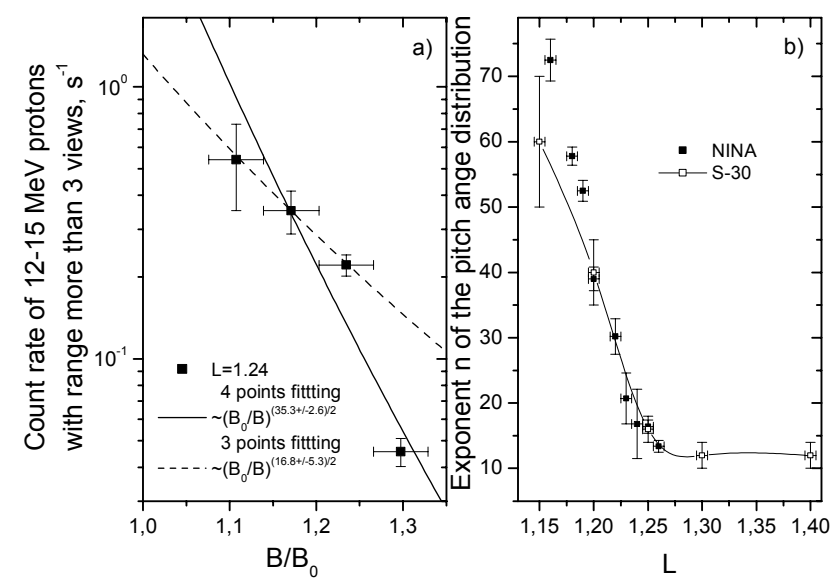

Fig. 3. (a) Counting rates of $12-15 \mathrm{MeV}$ protons with a range more than 3 views at the geomagnetic shell $\mathrm{L}=1.24$ as a function of geomagnetic latitude $\mathrm{B} / \mathrm{B}_{0}$. (b) The exponent $\mathrm{n}$ of the equatorial pitch angle distribution as a function of the L-shell value from the data of the NINA and S-30 experiments (Fischer et al., 1977).

density and the ensuing $\mathrm{n}$ parameter values at $\mathrm{L}<1.25$ are similar. The described method to calculate the exponent $n$ of pitch angle distribution is valid whenever the Liouville theorem is applicable. Near the loss cone, $\mathrm{n}$ is not constant, and direct measurements of pitch angle distribution are useful to validate physical models of angular distribution that takes the atmospheric properties into account (Heckman and Nakano, 1969).

In order to receive energy dependence of proton fluxes in the inner radiation belt, we used the data obtained in LT 

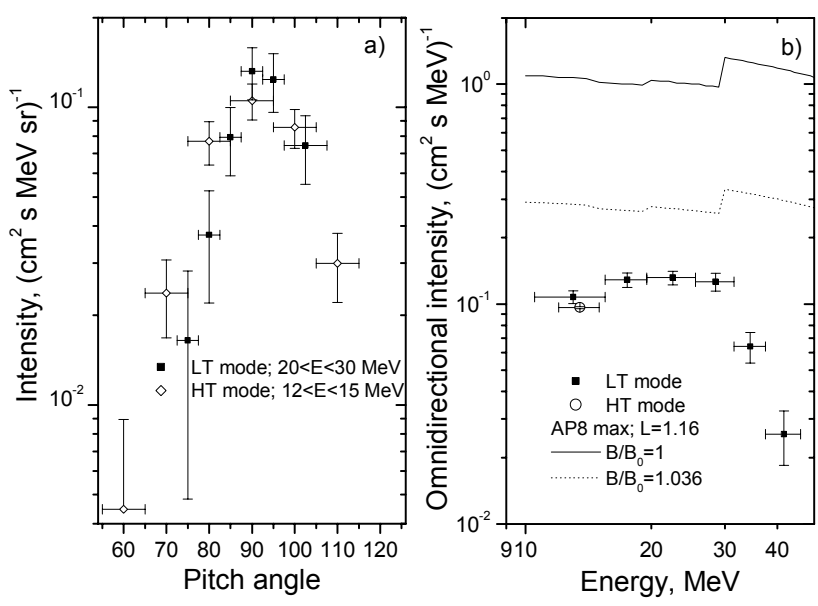

Fig. 4. (a) Equator pitch angle distributions of trapped protons at $\mathrm{L}=1.16$ for different energy ranges (12-15 MeV-open rhombus, 20$30 \mathrm{MeV}$ - solid squares). (b) Omnidirectional energy spectrum of trapped protons at $\mathrm{L}=1.16$ measured in different NINA operational modes (HT- open circle; LT- solid squares); AP-8 MAX predictions for $\mathrm{L}=1.16$ and for experimental $\mathrm{B} / \mathrm{B}_{0}$ range (solid line- $\mathrm{B} / \mathrm{B}_{0}=1$; dotted line- $\mathrm{B} / \mathrm{B}_{0}=1.036$ ).

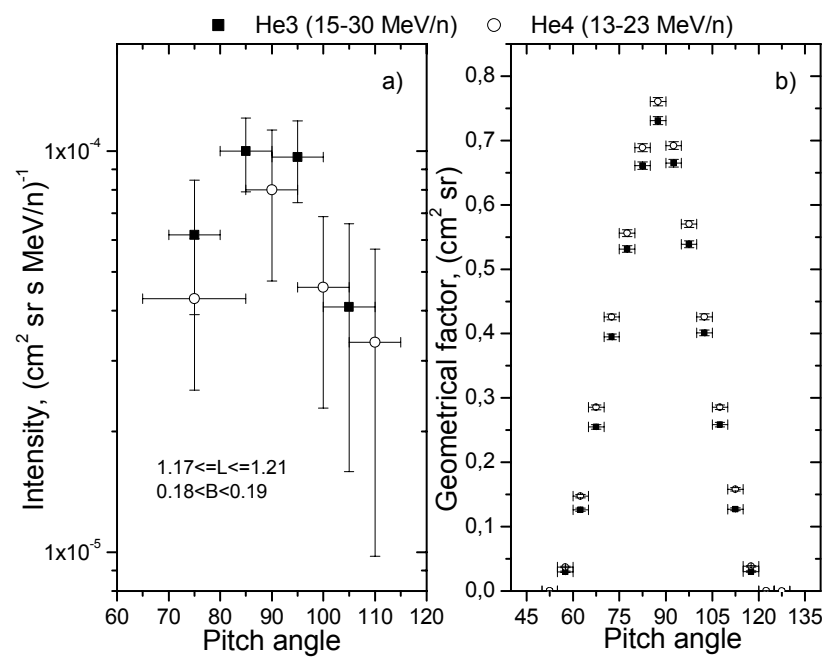

Fig. 5. (a) Pitch angle distributions of trapped ${ }^{3} \mathrm{He}$ (solid squares) and ${ }^{4} \mathrm{He}$ (open circles) isotopes in energy ranges $15-30 \mathrm{MeV} / \mathrm{n}$ and 13-23 MeV/n, correspondingly, for 1.17-1.21 L-bin and 0.18-0.19 B-bin. (b) Special NINA ${ }^{3} \mathrm{He}$ (solid squares) and ${ }^{4} \mathrm{He}$ (open circles) instrument geometrical factors as a function of particle pitch angle for orientation angle $\gamma=87.5^{\circ}$, which were used to reconstruct the pitch angle distributions.

mode. Pitch angle distributions for different energy ranges and the omnidirectional energy spectrum of trapped protons at L-shell 1.16 are shown in Figs. 4a and b, respectively. The predictions of AP-8 MAX model (http://www.spenvis.oma. be) for the experimental $\mathrm{B} / \mathrm{B}_{0}$ range $(1 ; 1.036)$ are also presented (Fig. 4b). The experimental spectrum is significantly steeper than the AP8 predictions. It is known that the AP-8 MAX fluxes at low energies and low $\mathrm{L}$ values are higher than the actual measurements (Hovestadt et al., 1972).

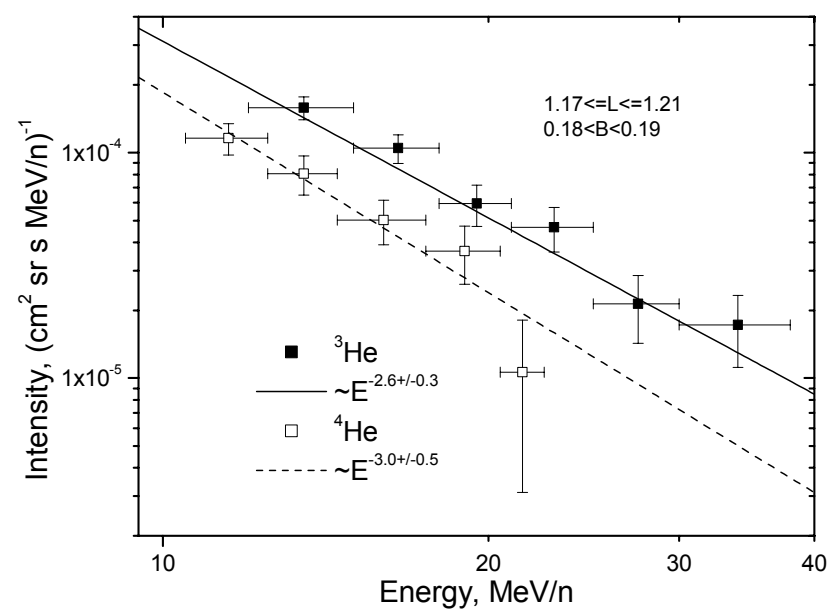

Fig. 6. Energy spectrum of trapped ${ }^{3} \mathrm{He}$ (solid squares) and ${ }^{4} \mathrm{He}$ (open squares) isotopes in 1.17-1.21 L-bin and 0.18-0.19 B-bin. The calculated spectra of trapped ${ }^{3} \mathrm{He}$ (solid curves) and ${ }^{4} \mathrm{He}$ (dotted curves) isotopes at L-shell 1.2 are also presented in Fig. 6. Upper curves correspond to the equator pitch angle value $80^{\circ}$ and the lower curves to $66^{\circ}$.

\subsection{Pitch angle distributions of trapped helium isotopes}

Due to the isotope identification ability of the NINA instrument, we could measure the isotope composition of the helium radiation belt component (Bakaldin et al., 2002a). It is believed that this component results from the nuclear interaction of primary radiation and the upper atmosphere near the inner edge of the inner radiation belt $(\mathrm{L}<1.3)$ (Pugacheva et al., 1998; Selesnick and Mewaldt, 1996; Galper et al., 2003). In this region ${ }^{3} \mathrm{He}$ isotope is more abundant than ${ }^{4} \mathrm{He}$, from energies more than $10 \mathrm{MeV} / \mathrm{n}$. We received pitch angle distribution of ${ }^{3} \mathrm{He}$ and ${ }^{4} \mathrm{He}$ isotopes (Fig. 5a) for energy ranges $15-30 \mathrm{MeV} / \mathrm{n}$ and $13-23 \mathrm{MeV} / \mathrm{n}$, correspondingly, using a calculated special geometrical factor presented in Fig. 5 b. Because of the low helium abundance in the inner radiation belt we needed to combine the data from the 1.17-1.21 L-bin and the $0.18-0.19 \mathrm{~B}$-bin. The energy spectra of He isotopes for the same $(\mathrm{B}, \mathrm{L})$ bin is shown in Fig. 6 . The ${ }^{3} \mathrm{He}$ spectrum is fitted by a power law with an index equal to $2.6 \pm 0.3$ in the energy range $12-40 \mathrm{MeV} / \mathrm{n}$, while ${ }^{4} \mathrm{He}$ has a spectral index of $3.0 \pm 0.5$ in the range $10-25 \mathrm{MeV} / \mathrm{n}$. These indexes are coincided with the values previously received for a more broad (B, L) bin (Bakaldin et al., 2002a). The calculated spectra of trapped ${ }^{3} \mathrm{He}$ (solid curves) and ${ }^{4} \mathrm{He}$ (dotted curves) isotopes at L-shell 1.2 are also presented in Fig. 6. Upper curves correspond to the equator pitch angle value $80^{\circ}$ and the lower curves to $66^{\circ}$. In the NINA experiment more than $70 \%$ of the events at L-shell 1.2 had as equator pitch-angle in this range (Galper et al., 2003). The calculations were done assuming a secondary nature of trapped helium isotopes in the inner radiation belt. The theory of secondary trapped helium production gives sufficient consent with the experimental values of helium intensities and for the ${ }^{3} \mathrm{He}^{4} \mathrm{He}$ ratio (Galper et al., 2003). 
It is necessary to also mention the ability of the NINA detector to measure full trapped particle angle distributions for validation of angular distribution models.

\section{Conclusions}

The capability of the NINA instrument in the direct measurement of the angular distribution of $\mathrm{H}$ and $\mathrm{He}$ isotopes has been described through the results acquired during a sixmonth operational period. The experimental direct measurements of trapped particle spectra and pitch angle distributions shows good agreement with the previous data, where they exist. The new measurements of trapped helium isotope pitch angle distribution are offered. On the other hand, the limitations of the instrument have been presented, in particular when it comes to data acquisition in the SAA.

The experimental results for the trapped helium isotope flux are in good agreement with calculations presented in Galper et al. (2003). These calculations show a sharp flux dependence from the equator pitch angle of trapped particles in the inner radiation belt, so the new detailed measurements of the trapped particles angle distributions are claimed.

Acknowledgements. We acknowledge the independent International Association formed by the European Community, European Union's Member states (INTAS), grant 03-55-1126 for partially support.

The COST 724 contributions to this publication are acknowledged. Topical Editor T. Pulkkinen thanks G. Esposito and E. Fiandrini for their help in evaluating this paper.

\section{References}

Armstrong, T. W., Colborn, B. L., and Watts, J. W.: Characteristics of trapped proton anisotropy at space station freedom altitudes, Science Applications International Corporation report SAIC-90/1474, 1990.

Auschrat, V. W. and Fischer, H. M.: Angular resolution of particle experiments on board spacecrafts without magnetic stabilisation, Nucl. Instrum. Methods, 122, 499, 1974.
Badhwar, G. D. and Konradi, A.: Conversion of omnidirectional proton fluxes into a pitch angle distribution, J. Spacecraft and Rockets 27, 350, 1990.

Bakaldin, A., Galper, A., Koldashov, S., et al.: Geomagnetically trapped light isotopes observed with the detector NINA, J. Geophys. Res., 107(A8), 81, 2002a.

Bakaldin, A., Galper, A., Koldashov, S., et al.: Light isotope abundances in solar energetic particles measured by the space instrument NINA, The Astrophysical Journal, 577, 513-523, $2002 \mathrm{~b}$.

Bidoli, V., Canestro, A., Casolino, M., et al.: In-orbit performances of the space telescope NINA and GCR flux measurements, Astrophys. J., Suppl. Ser., 132, 365-375, 2001.

Brun, R., Bruyant, F., Marie, M., et al.: GEANT detector description and simulation tool, W5014, Comput. and Networks Div., Eur. Org. for Nucl. Res., Geneva, Switzerland, 1994.

Cyamukungu, M., Grgoire, G., Heynderickx, D., et al.: Proton spectra detected by the proton switches on the CRRES satellite, J. Spacecraft and Rockets, 38, 4, 584-589, 2001.

Fisher, H. M., Auschrat, V. M., and Wibberenz G.: Angular distribution and energy spectra of protons of energy $5 \leq \mathrm{E} \leq 50 \mathrm{MeV}$ at the lower edge of the radiation belt in equatorial latitudes, J. Geophys. Res., 82, 4, 537, 1977.

Galper, A. M., Kodashov, S. V., Leonov, A. A., et al.: Light isotopes generation in the earth radiation belt, Izvestiy RAN. Ser. Phys. (in Russian), 67 (4), 525, 2003.

Heckman, H. H. and Nakano, G. H.: Low-altitude trapped protons during solar minimum period, 1962-1966, J. Geophys. Res., 74, $3575,1969$.

Hovestadt, D., Achtermann, E., Ebel, B., Hausler, B., and Pachmann, G.: New observations of the proton population of the radiation belt between 1.5 and $104 \mathrm{MeV}$, in Earth's Magnetospheric Processes, edited by: McCormac, B. M., D. Reidel Publishing Company, Dordrecht, Holland, 115, 1972.

Pugacheva, G. I., Spjeldvik, W. N., Gusev, A. A., et al.: Hydrogen and helium isotope inner radiaton belts in the Earth's magnetosphere, Ann. Geophys., 16, 931-939, 1998,

SRef-ID: 1432-0576/ag/1998-16-931.

Selesnick, R. S. and Mewaldt, R. A.: Atmospheric production of radiation belt light isotopes, J. Geophys. Res., 101, 9, 1974519757, 1996. 